\title{
Chapter 6 \\ Radiation Survey Along Two Trails in Mt. Fuji to Investigate the Radioactive Contamination Caused by TEPCO's Fukushima Daiichi Nuclear Plant Accident
}

\author{
Kazuaki Yajima, Kazuki Iwaoka, and Hiroshi Yasuda
}

\begin{abstract}
Mt. Fuji is located approximately $300 \mathrm{~km}$ southwest of TEPCO's Fukushima Daiichi Nuclear Power Plant. We performed the radiation survey along two main Mt. Fuji's trails from 04:30 to 18:30 on July 9, 2011. We climbed Yoshida trail from Fuji-Subaru Line fifth station $(2,300 \mathrm{~m}$ in altitude) to Yoshida-Subashiri top $(3,720 \mathrm{~m}$ in altitude) and descended Subashiri trail from the Yoshida-Subashiri top to Subashiri new fifth station $(2,000 \mathrm{~m}$ in altitude) on foot while measuring gamma rays. The dose rate $1 \mathrm{~m}$ above ground as measured using a $\mathrm{NaI}(\mathrm{Tl})$ scintillation survey meter was within the range from 0.03 to $0.05 \mu \mathrm{Sv} / \mathrm{h}$ throughout our measuring trip. We used a $\mathrm{NaI}(\mathrm{Tl})$ scintillation spectrometer for gamma-ray pulse height spectra measurement. The gamma-ray pulse height spectra obtained at least 2,500 $\mathrm{m}$ in altitude on both Yoshida and Subashiri trails showed the peaks of two radioactive cesium isotopes $\left({ }^{134} \mathrm{Cs}\right.$ and $\left.{ }^{137} \mathrm{Cs}\right)$ and natural radioactive nuclides. It was confirmed that a radioactive plume released from the Fukushima Daiichi Nuclear Power Plant had arrived at least 2,500 $\mathrm{m}$ above sea level of Mt. Fuji.
\end{abstract}

Keywords Dose rate $\bullet$ Plume $\bullet$ Radiation survey $\bullet$ Radioactive contamination

K. Yajima $(\bowtie) \cdot K$. Iwaoka

National Institute of Radiological Sciences,

4-9-1, Anagawa, Inage-ku, Chiba 2638555, Japan

e-mail: k_yajima@nirs.go.jp

H. Yasuda

National Institute of Radiological Sciences,

4-9-1, Anagawa, Inage-ku, Chiba 2638555, Japan

United Nations Scientific Committee on the Effects of Atomic Radiation,

Vienna International Centre, PO Box 500, Vienna 1400, Austria 


\subsection{Introduction}

A large amount of radioactive materials was released in the environment as a result of the TEPCO Fukushima Daiichi Nuclear Power Plant (NPP) accident caused by the Great East Japan Earthquake that occurred on March 11, 2011. The spread of the radioactive contamination had become a concern just after the accident. Mt. Fuji, which is $3,776 \mathrm{~m}$ above sea level and the highest mountain in Japan, is located approximately $300 \mathrm{~km}$ southwest of TEPCO's Fukushima Daiichi NPP. Because an environmental radiation monitoring level in Shizuoka City, which is approximately $50 \mathrm{~km}$ southwest from Mt. Fuji, rose on March 15 and $21-22,2011$, it is estimated that the radioactive plume released by TEPCO's

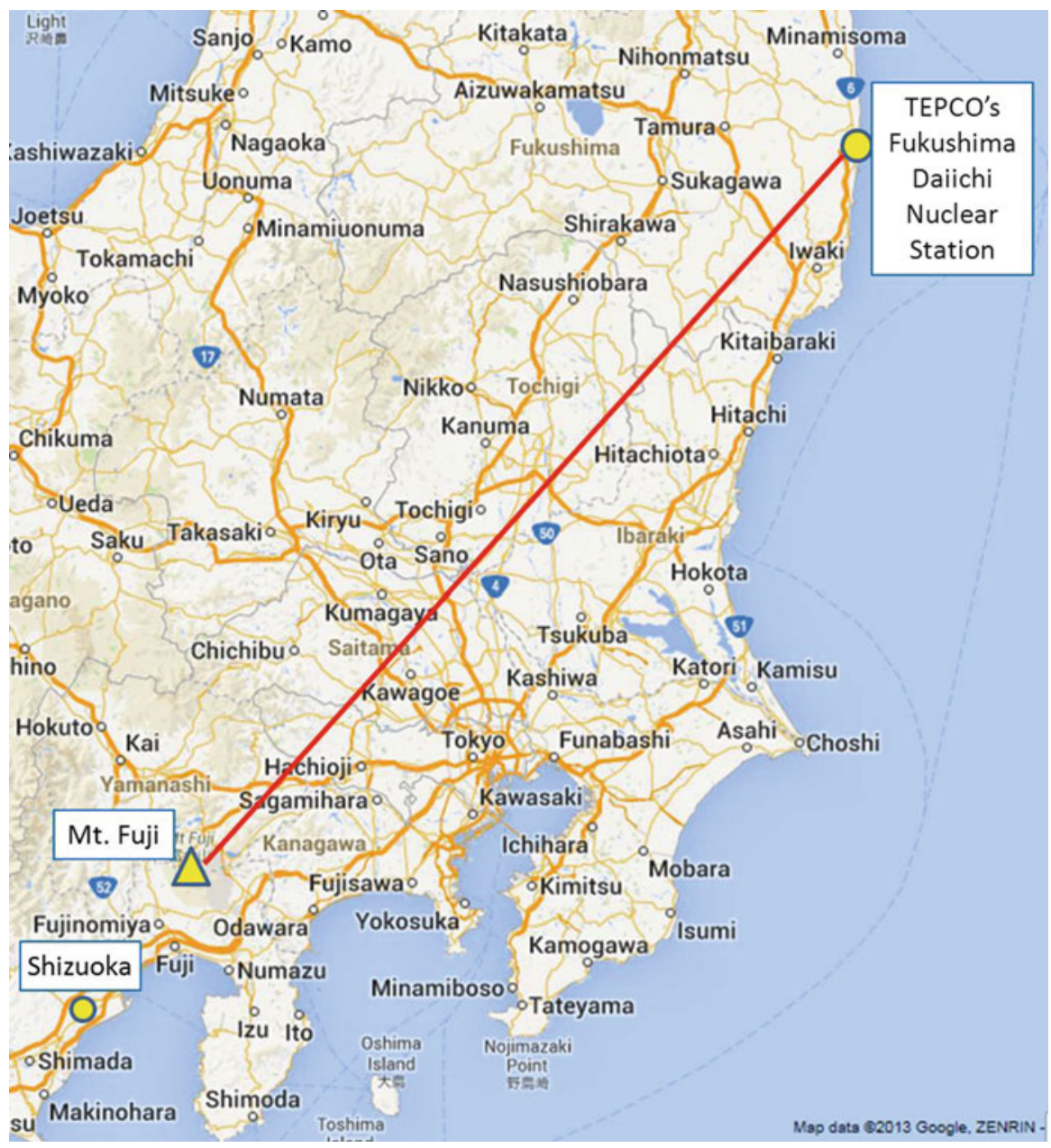

Fig. 6.1 Location of TEPCO's Fukushima Daiichi Nuclear Plant, Mt. Fuji, and Shizuoka City 
Fukushima Daiichi Nuclear Power Station arrived around Mt. Fuji at those times [1]. Figure 6.1 shows the locations of TEPCO's Fukushima Daiichi Nuclear Station, Mt. Fuji, and Shizuoka City.

Climbing Mt. Fuji is a very popular leisure activity and its trails are crowded with many climbers every summer. Thus, to reveal the radioactive contamination level of Mt. Fuji's trails before the summer season began, we performed the radiation survey along two main Mt. Fuji trails.

\subsection{Measurements}

The measurements were carried out from 04:30 to 18:30 on July 9, 2011. We climbed the Yoshida trail from the Fuji-Subaru Line fifth station 2,300 $\mathrm{m}$ above sea level to the top of Yoshida-Subashiri 3,720 m above sea level and descended Subashiri trail from the Yoshida-Subashiri top to the Subashiri-guchi new fifth station at 2,000 $\mathrm{m}$ above sea level on foot while measuring gamma-ray dose rate and pulse height spectra. A trace of our measuring trip at Mt. Fuji is shown in Fig. 6.2.

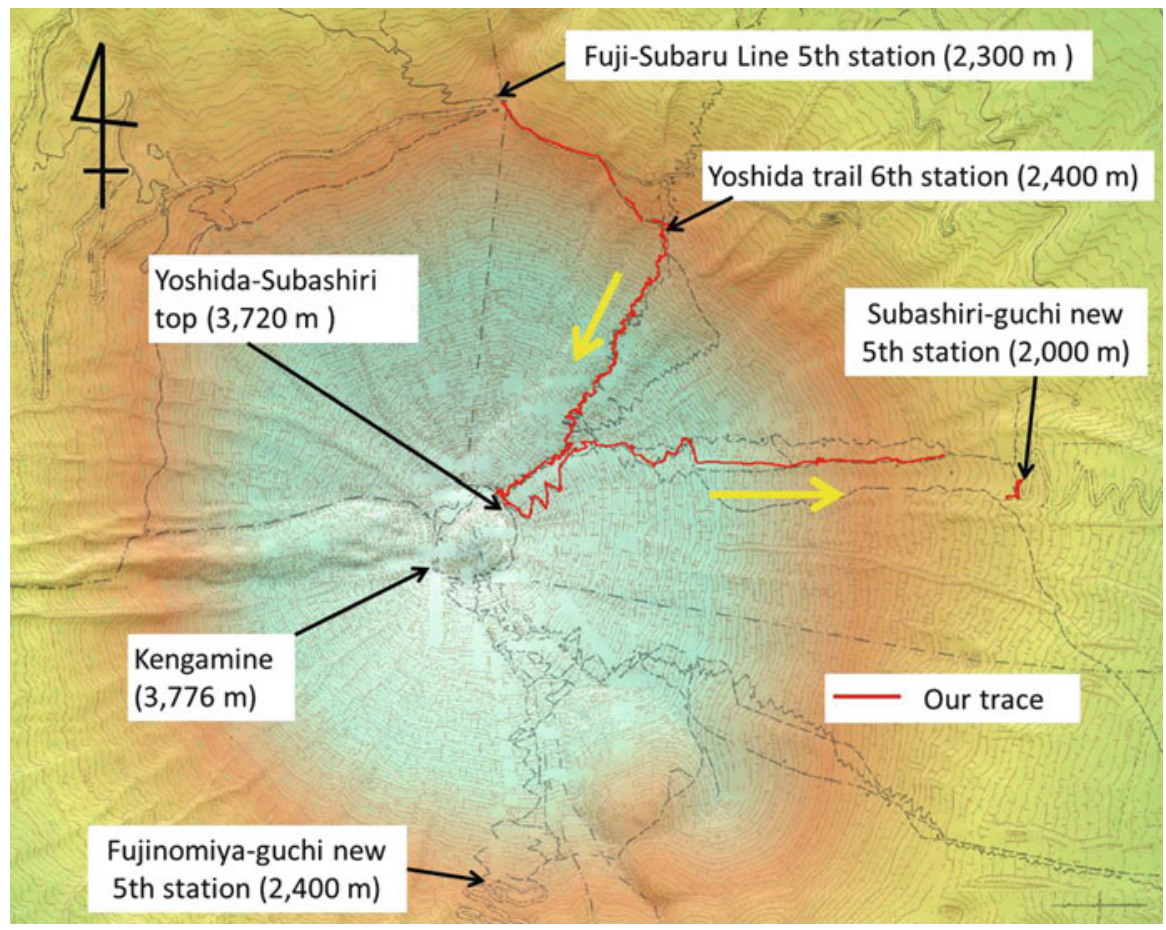

Fig. 6.2 A trace of our measuring trip at Mt. Fuji on July 9, 2011. We started from Fuji-Subaru line fifth station at 04:30 and reached Subashiri-guchi new fifth station at 18:30 


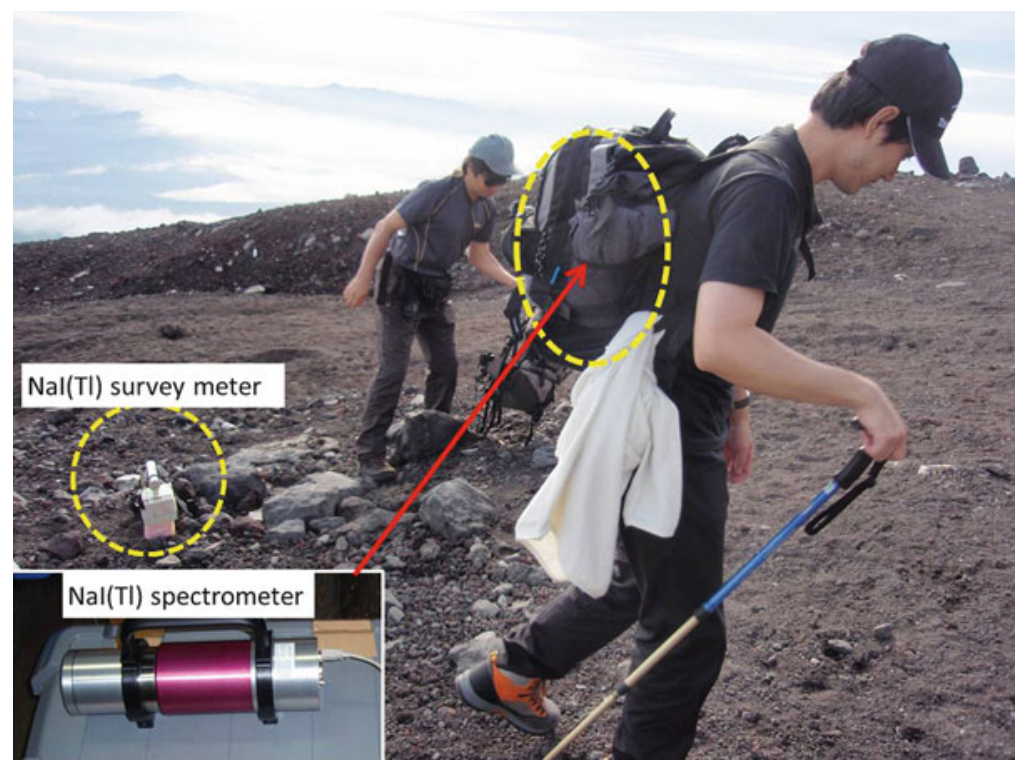

Fig. 6.3 Two of the authors on the measuring trip around Yoshida trail sixth station. The probe of the $\mathrm{NaI}(\mathrm{Tl})$ survey meter was set to an altitude of $1 \mathrm{~m}$ from the ground for dose rate measurements

The dose rate was measured using a $\mathrm{NaI}(\mathrm{Tl})$ scintillation survey meter (Aloka, TCS-172B; Hitachi Aloka Medical, Tokyo, Japan). The authors at times stopped moving and read the indicated values of the survey meter at a height of $1 \mathrm{~m}$ from the ground on the trail. A NaI(Tl) scintillation spectrometer (EMF211; EMF Japan, Osaka, Japan) with a tablet-size personal computer and an external power supply battery was used for gamma-ray pulse height spectrum measurements. The authors performed the 1-min repetition measurement, putting the spectrometer in a bag pack through the measuring trip. One scene of the measuring trip taken around the Yoshida trail sixth station 2,400 $\mathrm{m}$ above sea level is shown in Fig. 6.3.

\subsection{Results and Discussions}

The measured dose rates ranged from 0.03 to $0.05 \mu \mathrm{Sv} / \mathrm{h}$ throughout our measuring trip. As a result, it was found that the dose rates of the Yoshida trail and the Subashiri trail in Mt. Fuji were at nominal background levels. These two trails are located on the northeastern side of Mt. Fuji. There are two other major trails in Mt. Fuji. One is Fujinomiya trail, located on the south side of Mt. Fuji, and the other is Gotemba trail, on the southeastern side of Mt. Fuji. Arrival direction to Mt. Fuji and the horizontal expanse of the radioactive plume released from TEPCO's Fukushima Daiichi NPP have not been investigated yet. However, we assume that the dose rates of the Fujinomiya trail and Gotemba trail were in the same range (nominal background 
level) as the Yoshida and Subashiri trails because a difference in the degree of radioactive contamination is not seen around the Mt. Fuji area from the result of the airborne monitoring survey at an altitude less than 2,000 $\mathrm{m}$ by the Ministry of Education, Culture, Sports, Science and Technology (MEXT) [2].

Figure 6.4a-h shows the gamma-ray pulse height spectra given as the integration of the ten consecutive measurements (corresponding to 10-min collection time) measured at (a) the Fuji-Subaru Line fifth station, 2,300 m above sea level, (b) the Yoshida trail sixth station, $2,400 \mathrm{~m}$ above sea level, (c) the middle of the Yoshida trail, 2,500 $\mathrm{m}$ above sea level, (d) the middle of the Yoshida trail, 3,000 $\mathrm{m}$ above sea level, (e) the middle of the Yoshida trail, 3,400 m above sea level, (f) the YoshidaSubashiri top, 3,720 m above sea level, (g) the middle of the Subashiri trail, 2,500 m above sea level, and (h) the Subashiri new fifth station at 2,020 m above sea level, respectively. The spectra measured at less than $2,500 \mathrm{~m}$ above sea level showed the peaks of two radioactive cesium isotopes $\left({ }^{134} \mathrm{Cs}\right.$ and $\left.{ }^{137} \mathrm{Cs}\right)$ and natural radioactive nuclides. The peaks of ${ }^{134} \mathrm{Cs}$ and ${ }^{137} \mathrm{Cs}$ could not be identified in spectra that were measured more than 2,500 $\mathrm{m}$ above sea level.

It was confirmed that a radioactive plume released from TEPCO's Fukushima Daiichi NPP had arrived at least 2,500 $\mathrm{m}$ above sea level at Mt. Fuji. The ground surface is often above rain clouds on a high mountain such as Mt. Fuji. Therefore, we comment that the radioactive materials might hardly be deposited in the soil because it was dry, although the radioactive plume arrived at places more than 2,500 $\mathrm{m}$ above sea level. The airborne monitoring survey by MEXT has not provided data in mountain areas greater than $2,000 \mathrm{~m}$ in altitude because of difficulty in making flights. Therefore, it is hoped that our data become valuable information for the evaluation of the vertical distribution of the radioactive plume released by the Fukushima nuclear accident.

\subsection{Conclusion}

We performed a radiation survey along two main Mt. Fuji trails on July 9, 2011. The measured dose rates were within the range from 0.03 to $0.05 \mu \mathrm{Sv} / \mathrm{h}$ throughout our measuring trip, which were nominal background levels. From the results of these gamma-ray pulse height spectrum measurements, it was confirmed that a radioactive plume released from TEPCO's Fukushima Daiichi NPP had arrived at least 2,500 $\mathrm{m}$ above sea level at Mt. Fuji.

Acknowledgments The authors thank the staff of the NPO "Valid Utilization of Mt. Fuji Weather Station" for their kind advice about our Mt. Fuji climbing.

Open Access This article is distributed under the terms of the Creative Commons Attribution Noncommercial License which permits any noncommercial use, distribution, and reproduction in any medium, provided the original author(s) and source are credited. 

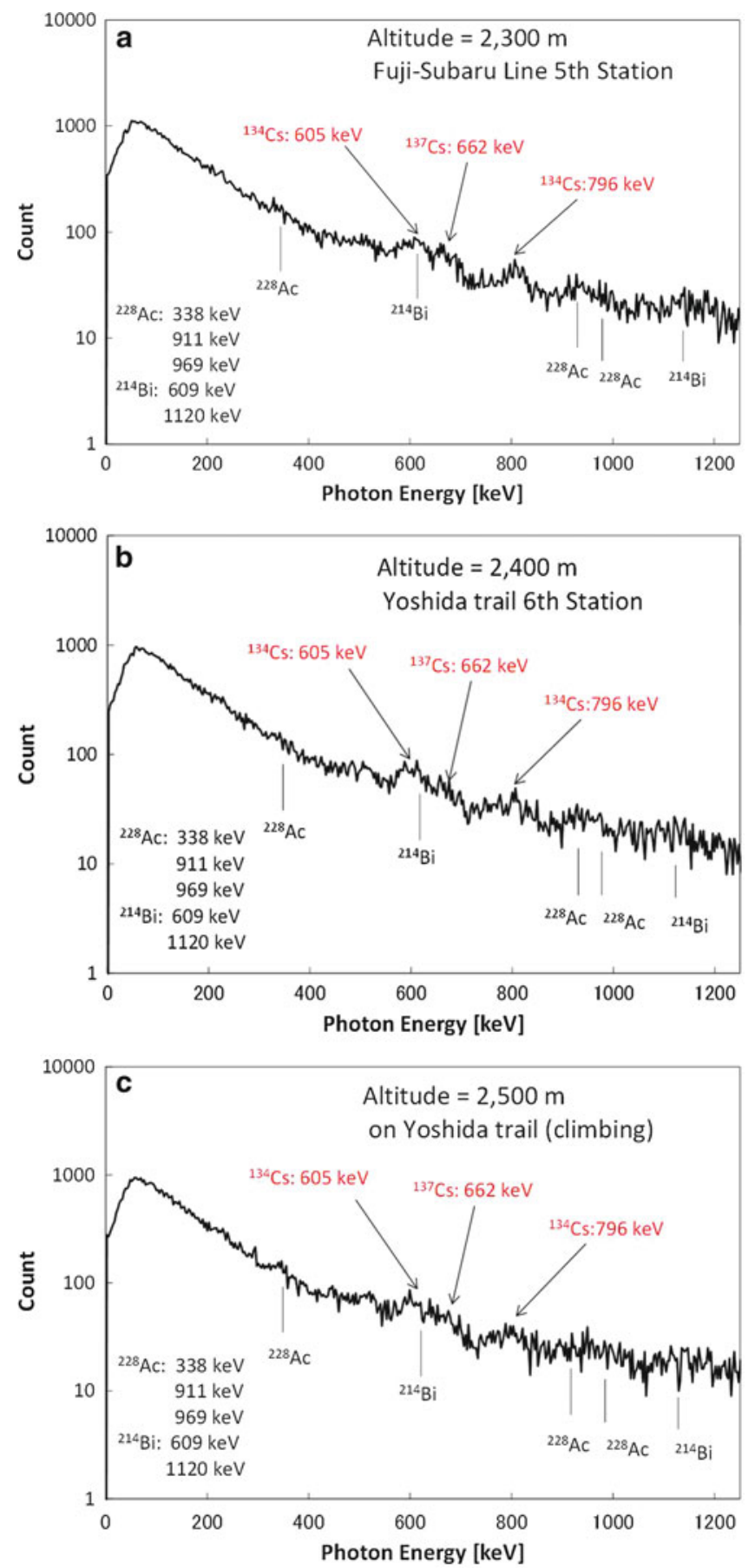

Fig. 6.4 a-h Gamma-ray pulse height spectra measured using a NaI(Tl) scintillation spectrometer on Mt. Fuji's trails 

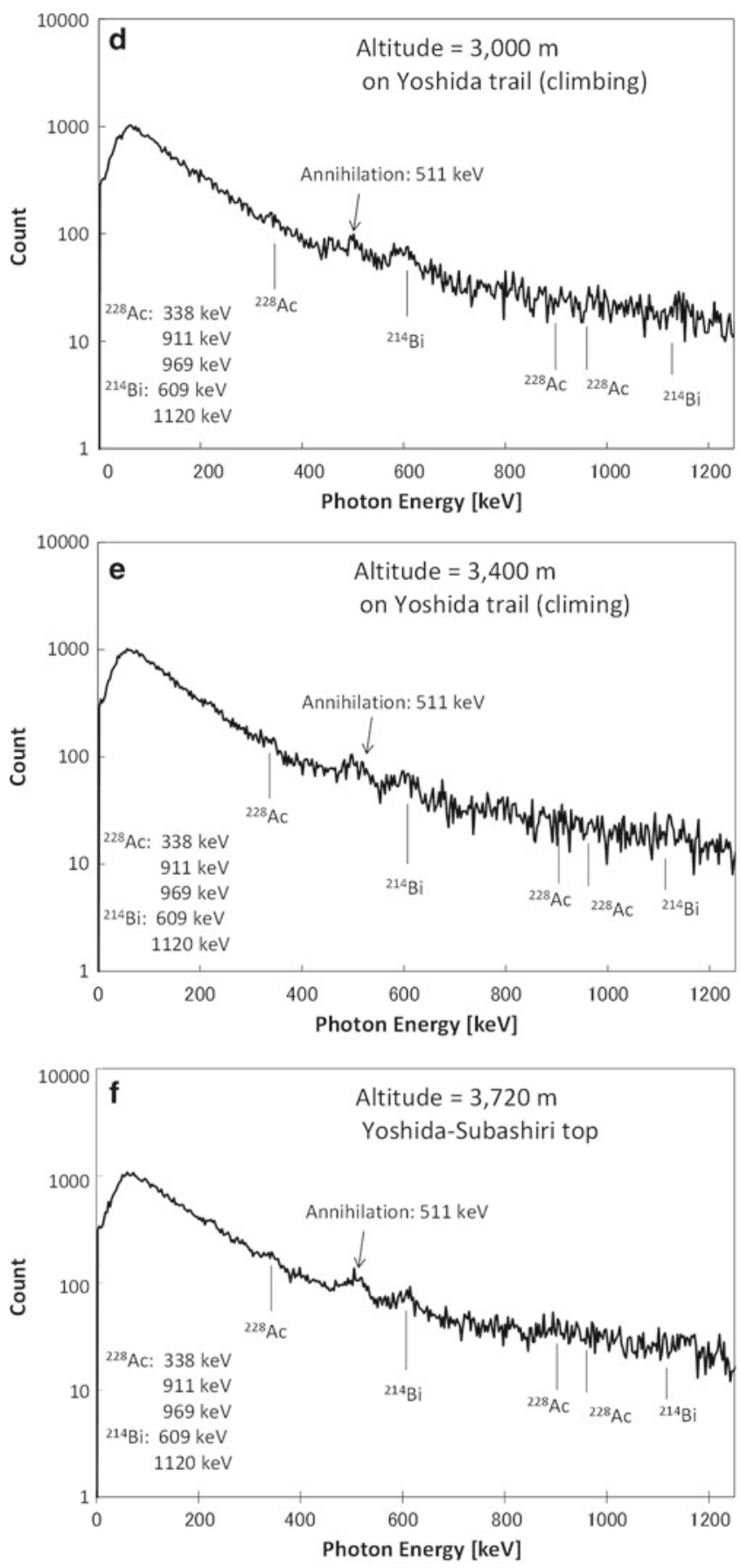

Fig. 6.4 (continued) 

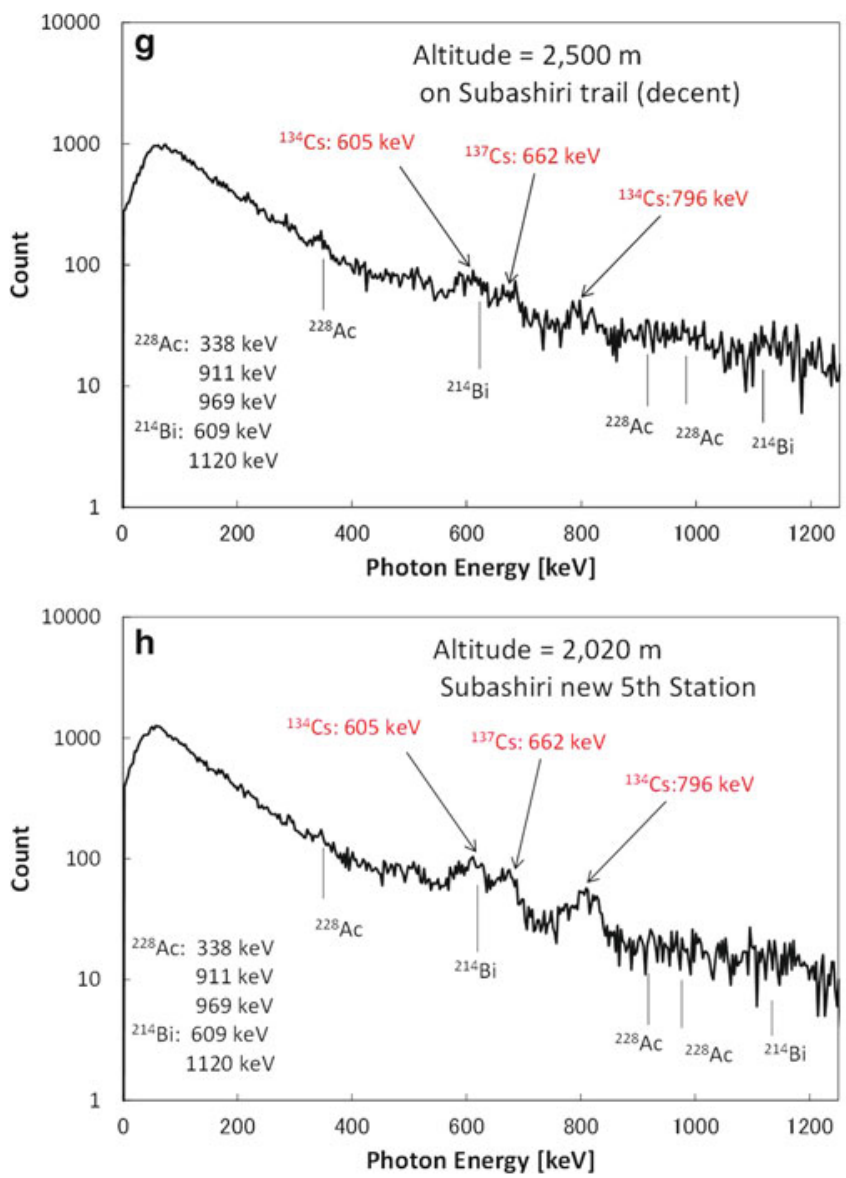

Fig. 6.4 (continued)

\section{References}

1. Shizuoka Prefecture website (in Japanese) (2013) http://www.pref.shizuoka.jp/ kinkyu/1fmonitoring/documents/110311-0831-1fruikei.pdf. Accessed 26 July 2013

2. Ministry of Education, Culture, Sports, Science and Technology (MEXT) (2013) Results of airborne monitoring survey by MEXT in Iwate, Shizuoka, Nagano, Yamanashi, Gifu, and Toyama Prefectures, and revision of the past airborne monitoring results by reflecting the influences of natural radionuclides. http://radioactivity.nsr.go.jp/en/contents/4000/3177/24/1270_111114.pdf. Accessed 26 July 2013 